\title{
TEST-RETEST RELIABILITY OF THREE-DIMENSIONAL GAIT ANALYSIS IN CHRONIC LOW BACK PAIN INDIVIDUALS: A PRELIMINARY STUDY
}

\author{
R. Fernandes (1,2), V. Moniz-Pereira (1), A. P. Veloso (1), P. A. Armada-da-Silva (1) \\ (1) CIPER - LBMF - Faculdade de Motricidade Humana - Universidade de Lisboa, Lisboa, Portugal \\ (2) Departamento de Fisioterapia, Escola Superior de Saúde - Instituto Politécnico de Setúbal, Setúbal, Portugal
}

Main topics: Reliability and service development: Analysis of clinical movement data

\section{INTRODUCTION and AIM}

Three-dimensional (3D) gait analysis is commonly used in research and clinical settings. Nevertheless, there are numerous sources of variability affecting the testing procedure (instrumental errors, anatomical landmark misplacement and soft tissue artifacts) [1], as well as inherent variability in gait performance, particularly in pathological gait. Few quality studies have been conducted regarding test-retest reliability in chronic low back pain (CLBP) individuals. Thus, the aim of this study was to investigate test-retest reliability of 3D gait analysis in a sample of chronic low back pain (CLBP) individuals.

\section{PATIENTS/MATERIALS and METHODS}

A prospective test-retest study design was conducted with a convenience sample of 8 CLBP individuals (43.8 \pm 6.7 yrs; $69 \pm 15.5 \mathrm{~kg} ; 164.9 \pm 8.4 \mathrm{~cm})$. All participants underwent two assessment sessions with an interval of 6 to 9 days. Each session included pain intensity (Numerical Rating Scale), disability (Quebec Back Pain Disability Scale), kinesiophobia (Tampa Scale of Kinesiophobia), and gait assessments. The gait data collection was carried out using a 13-camera opto-electronic system (Oqus 300, Qualisys AB, Gothenburg, Sweden) at 200Hz. Participants were instructed to walk during a few minutes at their preferred velocity and 10 gait cycles were selected to be processed in Visual 3D software (v5.01.10, C-Motion, Inc). A GCVSPL filter was applied to kinematic data. The marker set selection was based on previous reports [2] and a 9 segments' model (feet, shanks, thighs, pelvis, lumbar and thoracic spine) was built and optimized through global optimization [3]. Peak values for lower limb and trunk joint angles were computed for each trial and averaged for each subject. Intraclass Correlation Coefficient (ICC 2,1$)$ and their $95 \%$ confidence intervals for the 2-way random-effects model were calculated (IBM SPSS Statistics 20, p<.05). Standard error of the measurement (SEM) and minimal detectable change (MDC) were also computed for each variable according to a previous study [4].

\section{RESULTS}

There were no statistically significant differences in pain intensity, disability, kinesiophobia and anthropometric variables between the two assessment sessions. Reliability of peak values (maximum and minimum) for joint angles was examined and the results are shown on table 1. The obtained ICC values show high reliability to all parameters, very low SEM values $\left(<1^{\circ}\right)$ and very low MDC values $\left(<1^{\circ}\right.$, except to right hip peak flexion).

\section{DISCUSSION and CONCLUSIONS}

The results of this study show excellent values of test-retest reliability for lower limb and trunk kinematics during gait in CLBP individuals, together with a clinical acceptable level of error. These results also demonstrate that a very low amount of change would be sufficiently greater than measurement error, supporting the use of this method in clinical assessments of patients' gait patterns.

This work was funded by FCT through CIPER and SFRH/PROTEC/67505/2010.

\section{REFERENCES}

[1] Cereatti, A. et al. J Neuroeng Rehabil, 3:7, 2006.

[2] Seay, J et al. Sports Sci, 26(14):1519-29, 2008

[3] Lu, T.W. et al, J Biomech, 32:129-134, 1999.

[4] Meldrum, D. et al. Gait \& Posture, 33(1): 39,265-271, 2014.

Table 1: Reliability of thorax, lumbar and hips joints peak angles over gait cycle

$\begin{array}{lcccc}\quad \text { Kinematic Parameters } & \text { ICC } & \mathbf{9 5 \%} \mathbf{C l} & \text { SEM }^{\circ} & \text { MDC }^{\circ} \\ \text { Left Hip Angles } & & & & \\ \text { Peak Flexion } & 0.88 & 0.42-0.98 & 0.10 & 0.27 \\ \text { Peak Abduction } & 0.93 & 0.67-0.99 & 0.07 & 0.20 \\ \text { Peak External Rotation } & 0.87 & 0.33-0.97 & 0.16 & 0.45 \\ \text { Peak Extension } & 0.94 & 0.72-0.99 & 0.09 & 0.24 \\ \text { Peak Aduction } & 0.97 & 0.85-0.99 & 0.04 & 0.11 \\ \text { Peak Internal Rotation } & 0.90 & 0.55-0.98 & 0.13 & 0.37 \\ \text { Lumbar Angle } & & & & \\ \text { Peak Flexion } & 0.74 & -0.05-0.95 & 0.09 & 0.25 \\ \text { Peak Left Lateral Bending } & 0.91 & 0.59-0.98 & 0.06 & 0.18 \\ \text { Peak Left Rotation } & 0.85 & 0.26-0.97 & 0.03 & 0.09 \\ \text { Peak Extension } & 0.66 & -0.34-0.93 & 0.08 & 0.24 \\ \text { Peak Right Lateral Bending } & 0.78 & -0.06-0.96 & 0.09 & 0.24 \\ \text { Peak Right Rotation } & 0.83 & 0.14-0.97 & 0.05 & 0.13\end{array}$

\begin{tabular}{lcccc}
\multicolumn{1}{l}{ Kinematic Parameters } & ICC & $\mathbf{9 5 \%} \mathbf{C I}$ & SEM $^{\circ}$ & MDC $^{\circ}$ \\
Right Hip Angles & & & & \\
Peak Flexion & 0.84 & $0.19-0.97$ & 0.48 & 1.32 \\
Peak Abduction & 0.86 & $0.37-0.97$ & 0.17 & 0.47 \\
Peak External Rotation & 0.89 & $0.49-0.98$ & 0.29 & 0.80 \\
Peak Extension & 0.92 & $0.65-0.98$ & 0.08 & 0.23 \\
Peak Aduction & 0.88 & $0.44-0.98$ & 0.09 & 0.25 \\
Peak Internal Rotation & 0.93 & $0.68-0.99$ & 0.12 & 0.33 \\
Thorax Angles & & & & \\
Peak Flexion & 0.91 & $0.59-0.98$ & 0.07 & 0.20 \\
Peak Left Lateral Bending & 0.90 & $0.51-0.98$ & 0.10 & 0.27 \\
Peak Left Rotation & 0.81 & $0.10-0.96$ & 0.14 & 0.39 \\
Peak Extension & 0.94 & $0.72-0.99$ & 0.07 & 0.18 \\
Peak Right Lateral Bending & 0.91 & $0.59-0.98$ & 0.07 & 0.21 \\
Peak Right Rotation & 0.81 & $0.12-0.96$ & 0.12 & 0.33
\end{tabular}

